\title{
A new computer program for mass screening of visual defects in preschool children
}

\author{
D Briscoe, T Lifshitz, M Grotman, A Kushelevsky, H Vardi, S Weizman, B Biedner
}

\begin{abstract}
Aims-To test the effectiveness of a PC computer program for detecting vision disorders which could be used by nontrained personnel, and to determine the prevalence of visual impairment in a sample population of preschool children in the city of Beer-Sheba, Israel.

Methods-292 preschool children, aged 4-6 years, were examined in the kindergarten setting, using the computer system and "gold standard" tests. Visual acuity and stereopsis were tested and compared using Snellen type symbol charts and random dot stereograms respectively. The sensitivity, specificity, positive predictive value, negative predictive value, and kappa test were evaluated. A computer pseudo Worth four dot test was also performed but could not be compared with the standard Worth four dot test owing to the inability of many children to count.
\end{abstract}

Results-Agreement between computer and gold standard tests was $83 \%$ and $97.3 \%$ for visual acuity and stereopsis respectively. The sensitivity of the computer stereogram was only $50 \%$, but it had a specificity of $98.9 \%$, whereas the sensitivity and specificity of the visual acuity test were $81.5 \%$ and $83 \%$ respectively. The positive predictive value of both tests was about $63 \%$. $27.7 \%$ of children tested had a visual acuity of $6 / 12$ or less and stereopsis was absent in $28 \%$ using standard tests. Impairment of fusion was found in $5 \%$ of children using the computer pseudo Worth four dot test.

Conclusions-The computer program was found to be stimulating, rapid, and easy to perform. The wide availability of computers in schools and at home allow it to be used as an additional screening tool by non-trained personnel, such as teachers and parents, but it is not a replacement for standard testing.

(Br f Ophthalmol 1998;82:415-418)

Faculty of Health Sciences, Ben-Gurion University of the

Negev, Beer-Sheba, Israel

H Vardi

S Weizman

Correspondence to: B Biedner, MD, Department of Ophthalmology, Soroka Medical Centre, PO Box 151, Beer-Sheba, Israel.

Accepted for publication 5 November 1997

The prevalence of visual impairment in preschool children is significant and yet many patients are not treated owing to failure in detection. Visual disorders include refractive error, amblyopia, strabismus, infantile or juvenile cataract, leucocoria, and congenital or infantile glaucoma. One survey in the USA revealed that at least $5 \%$ of youths between 12 and 17 years of age had strabismus, but that only $1.4 \%$ had ever received treatment. In addition, it was intimated that at most only
$21 \%$ of preschool children were screened for major treatable visual disorders. ${ }^{12}$

There are a number of reasons for the poor level of screening in the community: firstly, the difficulty of obtaining access to children in order to examine them for visual dysfunction; secondly, the lack of parent awareness regarding the necessity for treatment early on. Thirdly, funding of a mass screening and parent awareness programme requires professionally trained personnel to direct it to the entire community. Different ethnic backgrounds and language problems can be a significant barrier and must be considered when planning mass screening. Beer-Sheba, in this respect, provided an ideal sample population for a pilot study in that many immigrant children of various ethnic backgrounds were included. Fourthly, most forms of testing are not stimulating and the child loses interest rapidly reducing the effectiveness of the test. There is a broad consensus that the most practical solution to this problem is the performance of mass screening using simple rapid techniques. ${ }^{13-5}$

Although there is disagreement among professionals as to the methodology of screening, it is widely accepted today that testing of stereopsis together with visual acuity is probably the most effective method. We, therefore, developed a computer program which incorporated tests of visual acuity, together with a colour random dot stereogram. In addition, we included a pseudo Worth four dot test which consisted of pictures instead of circles.

Visual acuity testing is the most widely used screening test for children but when used alone, it has several disadvantages. It does not distinguish between amblyopia and simple refractive errors and does not detect strabismus or suppression. It also requires the use of occluders which may distract the child and decrease cooperation. In addition the large number of visual acuity tests available creates difficulties of standardisation for judging results. ${ }^{6}$

The random dot stereogram (RDS) has 
Table 1 Tests used

\begin{tabular}{lll}
\hline Variable & Computer system test & Gold standard test \\
\hline Visual acuity & Snellen pictures & Snellen pictures \\
Fusion and & $\begin{array}{l}\text { Pseudo Worth four dot test } \\
\text { stereopsis }\end{array}$ & $\begin{array}{l}\text { Worth four dot test (13 children only) } \\
\text { stereogram }\end{array}$ \\
& RDS “E” \\
\hline
\end{tabular}

Table 2 Prevalence of visual abnormalities in 292 children between 4 and 6 years of age in the Negev region

\begin{tabular}{|c|c|c|c|c|}
\hline \multirow[b]{2}{*}{ Abnormality } & \multicolumn{2}{|c|}{ No of children } & \multicolumn{2}{|l|}{ Prevalence } \\
\hline & $\begin{array}{l}\text { Standard } \\
(n=290)\end{array}$ & $\begin{array}{l}\text { Computer } \\
(n=292)\end{array}$ & Standard & Computer \\
\hline $\mathrm{VA} \leqslant 6 / 12$ & 81 & 102 & $27.7 \%$ & $34.9 \%$ \\
\hline$V A \leqslant 6 / 24$ & 14 & 11 & $3.8 \%$ & $4.8 \%$ \\
\hline Stereopsis & 8 & 10 & $2.8 \%$ & $3.4 \%$ \\
\hline \multicolumn{5}{|c|}{ Fusion (WFD) } \\
\hline Far & - & 15 & - & $5.1 \%$ \\
\hline Near & - & 3 & - & $1.0 \%$ \\
\hline
\end{tabular}

$\mathrm{VA}=$ visual acuity; WFD $=$ Worth four dot test.

the patient and is simple enough to be performed on children who can count to five.

We designed a computer program which is simple to use and allows rapid screening examinations by non-trained personnel. Computers are widely available in most schools and often also in the home, thus enabling accessibility of the program to most children. In addition, screening can be performed by teachers, parents, or volunteers. The aim of our pilot study was to determine the effectiveness of the computer program as an additional tool for mass screening of visual impairment compared with standard tests. We also aimed to estimate the prevalence of visual dysfunction at preschool age in the city of Beer-Sheba.

\section{Populations and methods}

In all, 292 children between the ages of 4 and 6 years, from seven kindergartens, were screened, using the computer system and "gold standard" tests. The system consists of a 386 IBM compatible computer, working under Windows with a 14 inch SVGA screen. The software used was developed by Optitest, Ofakim, Israel. The kindergartens were chosen randomly from the municipality register of kindergartens in the city of Beer-Sheba.

Each child was examined in a secluded area of the kindergarten where there were no distractions. The same computer hardware was used throughout the study. A number of days later each child was re-examined using the gold standard tests. At re-examination the previous results were not available in order to minimise examiner bias. The fact that the standard test was not performed on the same day as the computer test allowed for maximal concentration of children without distraction.

The tests used are described and summarised in Table 1.

\section{VISUAL ACUITY}

Visual acuity was measured using Snellen type pictures (symbol test) on the computer screen, with the child and the computer 3 metres in front of a mirror. These figures could be shown in a line in the usual way or separately with the differing figures getting progressively smaller.
The latter method was especially helpful in children with a poor concentration span. The standard test was performed in an identical fashion using a chart.

The mirror is not ideal for testing visual acuity as children may find it more difficult. It was, however, practical for the purpose of this pilot study where the aim was to access mass screening for children in an identical fashion, often in kindergartens without ideal conditions.

\section{STEREOPSIS}

Stereopsis was evaluated by the computed program using a colour based random dot stereogram which displayed pictures and shapes that were familiar to 4 year old children. The test was carried out at a $40 \mathrm{~cm}$ distance. Every third picture displayed was a circle, triangle, or star as these figures were best identified by the children. Children with absent stereopsis were disappointed and bored when they saw no "magic picture" and would lose interest quickly. We therefore added a function that drew the stereo image on the screen at the press of a button, and thus allowed them to see it. In this way, the child's interest was kept up without loss of motivation. The standard random dot E stereogram (RDES) was used at the $40 \mathrm{~cm}$ distance currently recommended. ${ }^{10}$

\section{WORTH FOUR DOT TEST}

The computer program's Worth four dot comprised the following shapes-two stars (green), one square (white), and one circle (red). This enabled the child to identify and communicate exactly what he saw-for example, a circle, a star, a star, and a square.

However, we experienced great difficulty with the gold standard test owing to the fact that it depends on the ability of the child to count. Many of the children in our study could not count, making it almost impossible to decide whether the test was normal or abnormal. On this basis, we discontinued performing the standard Worth four dot test. However, before examining with the pseudo Worth dot test, we performed a "quality control" test on every child by covering each eye to check that the filters worked.

At any point during all tests the children could be encouraged or rewarded by pressing a button which triggered fireworks and music to appear, enabling us to keep their attention and interest.

Statistical analysis was carried out using the standard tests and the following measurements were obtained: sensitivity, specificity, positive predictive value, negative predictive value, and kappa test, the definitions of which are outlined below.

Sensitivity shows the percentage of abnormal computer tests out of all abnormal gold standard tests.

Specificity measures the percentage of normal computed tests out of all normal gold standard tests.

Positive predictive value shows the likelihood that a child with an abnormal computer test will be really abnormal. 
Table 3 Summary of effectiveness of computer examination compared with standard test ("gold standard")

\begin{tabular}{lll}
\hline & Visual acuity tests (symbol) & Stereopsis (RDS) \\
\hline Percentage agreement & 83 & 97.3 \\
Percentage disagreement & 17 & 2.7 \\
Sensitivity & 81.5 & 50 \\
Specificity & 83 & 98.9 \\
Positive predictive value & 64.7 & 62.5 \\
Negative predictive value & 92.1 & 98.2 \\
Kappa & 0.6 & 0.54 \\
\hline
\end{tabular}

RDS = random dot stereogram.

Table 4 Percentage of disagreement between the two methods, in visual acuity testing, according to number of lines of difference

\begin{tabular}{lllll}
\hline No of lines difference & $\begin{array}{l}\text { No of } \\
\text { cases }\end{array}$ & $\begin{array}{l}\text { \% From 290 } \\
\text { children }\end{array}$ & $\begin{array}{l}\text { \% Underestimation } \\
\text { out of 290 children }\end{array}$ & $\begin{array}{l}\text { \% Overestimation } \\
\text { of 290 children }\end{array}$ \\
\hline 2 and 3 lines (total) & 25 & 8.6 & $1.7(5)$ & $6.9(20)$ \\
2 lines & 20 & 6.9 & $1.4(4)$ & $5.5(16)$ \\
3 lines & 5 & 1.7 & $0.3(1)$ & $1.4(4)$ \\
\hline
\end{tabular}

Negative predictive value shows the likelihood that a child with normal computer test will be normal.

The kappa test was used as a measure of agreement between methods. Interpretation of the kappa statistics is based on the following guidelines outlined by Landis and $\mathrm{Koch}^{13}$ :

0.20 slight agreement

$0.21-0.40$ fair agreement

$0.41-0.60$ moderate agreement

$0.61-0.80$ good agreement

0.81-1.00 almost perfect agreement.

\section{Results}

In all, 292 children between the ages of 4 and 6 years were examined. Of these, two children refused to undergo examination with standard tests so that 290 children were used for comparing the tests. The prevalence of visual abnormalities in the children examined is shown in Table 2. The prevalence of visual disturbance was higher according to the computer tests than it was actually found to be using standard tests.

The prevalence of impaired visual acuity was found to be $27.7 \%$ using standard Snellen type pictures and $34.9 \%$ using the computer program. Absent stereopsis was found in $2.8 \%$ of children using standard RDES and in 3.4\% using the computer stereogram. Five per cent of children examined had absent fusion using the computer pseudo Worth dot test.

A comparison of the effectiveness of the computer tests compared with standard tests is shown in Table 3.

There was good agreement between the computer tests and the gold standard tests although the percentage agreement was much higher between tests of stereopsis than it was between tests of visual acuity. Similarly, there was greater disagreement between visual acuity tests than there was between tests of stereopsis. The sensitivity of the computer stereogram, however, was lower than the sensitivity of the computer visual acuity test. The specificity of the computer tests was very good with a higher percentage specificity for the stereogram than for the visual acuity test. The positive predictive values of both the visual acuity test and the stereogram were similar although the negative predictive value of the computer stereogram was greater than that of the visual acuity test. The kappa value shows that this was not by chance.

In 25 cases out of 290 there was disagreement between the computer and the standard visual tests of two lines or more. Analysis of these cases is shown in Table 4. Of these 25 cases 20 had a two line difference and five had a three line difference.

In 20 of the 25 cases there was overestimation of visual impairment and in five cases there was underestimation. However, of the five latter cases where visual impairment was underestimated three of them were screened out by the computer stereogram and would therefore have been referred for treatment.

\section{Discussion}

Results of this pilot study show that the prevalence of abnormal visual acuity (6/12) among 4-6 year old children in the Negev region is $27.7 \%$ and that stereopsis was absent in $2.8 \%$ of children examined, using standard tests. Abnormal fusion was found in $5 \%$ of children examined with the pseudo Worth four dot test.

It has already been reported by Hammond and Schmidt, ${ }^{14}$ that refractive errors of up to +3.00 dioptres of hyperopia, $1.50 \mathrm{D}$ of astigmatism, and $2.0 \mathrm{D}$ of myopia were present in children who passed the RDES at a high threshold of 2 metres distance (168 seconds of arc). This would explain the large difference obtained in our pilot study between abnormal visual acuity and absent stereopsis where the RDES was performed at a low threshold $40 \mathrm{~cm}$ distance (638 seconds of arc). At the high threshold 2 metres distance Ruttum and Nelson ${ }^{10}$ reported that underreferral increased by $46 \%$ and they therefore recommended that the RDES be performed at a $40 \mathrm{~cm}$ distance only and as a complementary test to visual acuity.

In comparing the computer tests developed with gold standard tests there was a high percentage of agreement and specificity between them with positive predictive values in the region of $63 \%$. The kappa values obtained show that this was not by chance. Visual acuity testing showed disagreement in a total of 25 out of 290 cases $(8.6 \%)$ where there was at least a two line difference between tests. This is comparable with the results of Ruttum and Nelson ${ }^{10}$ and who found disagreement between the results of screeners and examiners on the same basis of a two line difference. However, of the 25 cases of disagreement in visual acuity testing, $80 \%$ overestimated visual dysfunction as opposed to the equal distribution of over and underestimations in the series of Ruttum and Nelson. ${ }^{10}$ Three out of five of the underestimated cases of visual dysfunction were screened out by the computer stereogram. This in effect reduced the number of underreferred cases by the computer program.

The specificity of the visual acuity test was similar to previous results of studies comparing screener and examiner testing using standard tests. The sensitivity of the computer stereogram was only $50 \%$ although the specificity was almost $99 \%$. This may be due to two 
factors. Firstly, the computer tests need to be performed in a dimly lit room in order to minimise interference with the light radiating from the television screen whereas the standard test depends on adequate and bright lighting in the examining area. Our study was carried out during the bright summer season making it difficult to darken kindergarten rooms sufficiently and thus working to the advantage of the standard tests. The second factor is a learning factor in that all standard tests were performed on the second visit after the computer tests. Thus, children knew what to expect the second time. In retrospect, it may have been better to first perform the standard tests in half of each kindergarten class tested.

Our pilot study found the prevalence of visual impairment at preschool age to be at least $27.7 \%$ in the Beer-Sheba municipality. Absent stereopsis was found in at least $2.8 \%$ of children examined. This computer program clearly does not replace standard testing, but it does present a useful, additional way of mass screening for visual impairments at preschool age by non-trained personnel.

1 Ehrlich MI, Reinecke RD, Simons K. Preschool vision screening for amblyopia and strabimsus. Programmes, methods, guidelines. Surv Ophthalmol 1983;28:145-63.
2 US National Center for Health Statistics. Eye examination findings among youths 12-17 years. Vital and Health Statistics Series 11, No 155. Washington DC: DHEW Pub No (HRA) 1976;76:1637

3 Reinecke R. Current concepts in ophthalmologystrabismus. N Engl f Med 1979;300:1139-41.

4 Newman DK, Hitchcock A, McCarthy H, Keast Butler J, Moore PT. Preschool vision screening outcome of children referred to hospital eye service. Br f Ophthalmol 1996;80: $1077-82$.

5 Wasserman R, Croft C, Brotherton S. Preschool vision screening in pediatric practice. Paediatrics 1992;89:834-8. 6 Simons K. Visual acuity norms in young children. Surv Ophthalmol 1983;28:84-92.

7 Simons K, Avery KE, Novak A. Small target random dot stereogram and binocular suppression testing for preschool vision screening. F Pediatr Ophthalmol Strabismus 1996;33: 104-13.

8 Hered RW, Murphy S, Clancy M. Comparison of the HOTV and Lea symbol charts for preschool vision screening. F Pediatr Ophthalmol Strabismus 1997;34:24-8.

9 Simons K. Stereoacuity norms in young children. Arch Ophthalmol 1981;99:439-45.

10 Ruttum MS, Nelson DB. Stereopsis testing to reduce overreferral in preschool vision screening. $\mathcal{F}$ Pediatr Ophthalmol Strabismus 1991;28:131-3.

11 Thompson JR, Woodruff G, Hiscox FA, Strong N, Minshull $\mathrm{C}$. The incidence and prevalence of amblyopia detected in C. The incidence and prevalence of amblyo
childhood. Public Health 1991;105:455-62.

12 Vinding T, Gregerson E, Jensen A, Rindziunski E. Prevalence of amblyopia in old people without previous screening and treatment. An evaluation of the present prophylactic procedures among children in Denmark. Acta Ophthalmol Copenh 1991;69:796-8.

13 Landis JR, Koch GG. An application of hierarchical kappatype statistics in the assessment of majority agreement among multiple observers. Biometrics 1977;33:363-74.

14 Hammond RS, Schmidt PP. A Random Dot E Stereogram for the vision screening of children. Arch Ophthalmol 1986; 104:54-60. 